\title{
Limitations of a simplified dangling bond recombination model for a-Si:H
}

\author{
Tsu-Tsung Andrew Li, ${ }^{1, a)}$ Keith R. Mclntosh, ${ }^{2}$ and Andres Cuevas ${ }^{1}$ \\ ${ }^{1}$ Department of Engineering, CECS, The Australian National University, Canberra ACT 0200, Australia \\ ${ }^{2}$ Centre for Sustainable Energy Systems, The Australian National University, Canberra ACT 0200, \\ Australia
}

(Received 23 July 2008; accepted 19 October 2008; published online 10 December 2008)

\begin{abstract}
The validity of a widely used simple closed-form expression for the recombination associated with dangling bonds in hydrogenated amorphous silicon $(a-\mathrm{Si}: \mathrm{H})$ is linked to the relative position of the distribution of the dangling bond states with respect to the quasi-Fermi levels for trapped electrons and holes. However, these quasi-Fermi levels for traps have not been derived before. In this work, we derive the four relevant quasi-Fermi levels for traps associated with dangling bonds in $a$-Si:H and clarify the limitations of the simple model. (C) 2008 American Institute of Physics.
\end{abstract}

[DOI: $10.1063 / 1.3037235$ ]

\section{INTRODUCTION}

Hydrogenated amorphous silicon $(a-\mathrm{Si}: \mathrm{H})$ contains an amphoteric dangling bond (DB) defect that can be neutral $\left(T_{3}^{0}\right)$, positively charged $\left(T_{3}^{+}\right)$, or negatively charged $\left.\left(T_{3}^{-}\right)\right)^{1,2}$ The defect's energy level depends on its charge state, and there are two energy levels for the DB in $a$-Si:H (see Fig. 1). Consequently, the recombination rate associated with the DB $\left(U_{\mathrm{DB}}\right)$ does not follow Shockley-Read-Hall ${ }^{3}$ (SRH) statistics or its extension developed by Simmons and Taylor ${ }^{4}$ to allow for a distribution of energy states. Instead, $U_{\mathrm{DB}}$ follows the statistics of correlated electrons, ${ }^{1,2,5}$ which is more complicated and requires knowledge of the defect density, as well as the emission and capture rates in each of the different energy levels.

Defects associated with DBs are the most important channel for carrier recombination in $a$-Si:H. An ability to understand and model this recombination is of extreme importance for fundamental studies of $a-\mathrm{Si}: \mathrm{H}$ as well as analyses of $a-\mathrm{Si}: \mathrm{H}$ semiconductor devices. However, most solutions are numerical and require the knowledge of many parameters, making them difficult to apply. Because of this, an approximate but simple closed-form solution for $U_{\mathrm{DB}}$ was derived by Hubin et al. ${ }^{6}$ and has become a popular choice among researchers. ${ }^{7-11}$ Unfortunately, this simple model has a limited range of validity and can be easily misused.

This simple closed-form solution $U_{\mathrm{DB} \text {.scf }}$ is indeed much easier to calculate than the general solutions for $U_{\mathrm{DB}}$ because it requires fewer parameters. Its validity, however, is subject to several assumptions, the most important being that all the DB states need to be recombination centers and not traps. In this context we refer to a trap as a relatively shallow energy level that interacts with the relevant band (conduction or valence) without leading to carrier recombination.

Unfortunately, Hubin et al. ${ }^{6}$ did not clarify the conditions that validate that key assumption. In particular, they did not distinguish demarcation levels ${ }^{12}$ from quasi-Fermi levels for traps. ${ }^{13}$ At the energy states defined as demarcation levels, the probability of an electron (or hole) recombining or

${ }^{a)}$ Electronic mail: tsu-tsung.li@anu.edu.au. being thermally re-emitted is equal, whereas within the quasi-Fermi levels for traps, the probability of an electron (or hole) being thermally re-emitted is negligible; the distinction between the two is explained in detail and presented diagrammatically by Simmons and Taylor. ${ }^{4}$ The quasi-Fermi levels for traps therefore provide a more appropriate distinction between trapping and recombination centers: energy levels between a pair of quasi-Fermi levels for traps behave as recombination centers, and outside them they behave as trapping centers. ${ }^{4}$ Therefore, the work presented in this paper uses them as the key criteria for validating the key assumption behind $U_{\mathrm{DB} . s c f}$.

A quasi-Fermi level for trapped electrons and a quasiFermi level for trapped holes can be derived for a particular species of defects, which is defined by a constant ratio of the capture cross sections for electrons and holes [i.e., $k$ $=\sigma_{n}(E) / \sigma_{p}(E)$, where $k$ is a constant]. In the general case there will be many species of defect levels, which will result in two corresponding quasi-Fermi levels for traps for each of the species. In the case of the $U_{\mathrm{DB} . s c f}$ where the capture cross sections are independent of energy, there are two species of defects and subsequently four quasi-Fermi levels for traps.

These four quasi-Fermi levels for traps have not been explicitly derived before: Hubin et $a l^{6}{ }^{6}$ only referred to Tay-

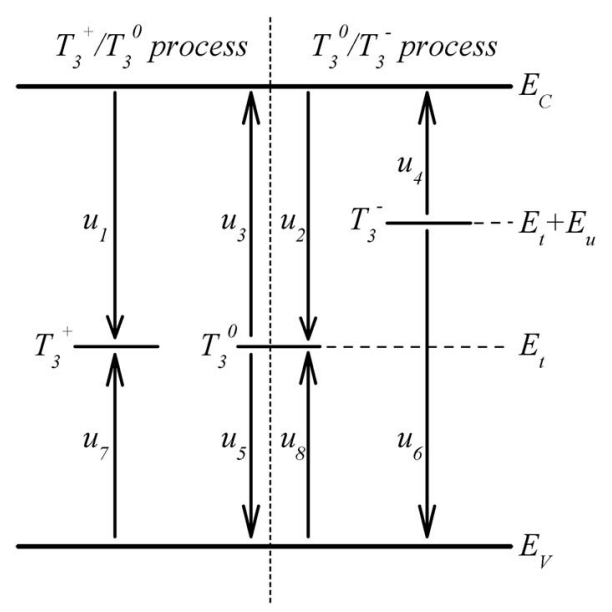

FIG. 1. Schematic representation of electron flows for the DB model. 
lor and Simmons, ${ }^{13}$ who in turn explained the general concept but only derived the relevant quasi-Fermi level for traps for SRH recombination but not for $U_{\mathrm{DB} . \mathrm{scf}}$.

In this paper we first apply DB recombination statistics to derive $U_{\mathrm{DB}}$ and then describe the key assumptions that permit the derivation of $U_{\mathrm{DB} . s c f}$. The four quasi-Fermi levels for traps are then derived and their application to evaluate the validity of the simple model is illustrated with representative examples.

\section{RECOMBINATION STATISTICS FOR $U_{\mathrm{DB}}$}

Figure 1 illustrates the DB recombination model as presented by Vaillant and Jousse. ${ }^{2}$ It contains two energy states $\left(E_{t}\right.$ and $\left.E_{t}+E_{u}\right)$, three charge states $\left(T_{3}^{+}, T_{3}^{0}, T_{3}^{-}\right)$, and eight carrier flows $\left(u_{1}-u_{8}\right)$. In this representation, $E_{t}$ and $E_{t}+E_{u}$ are discrete, but the following derivation permits a distribution of $E_{t}$ with energy.

To determine the recombination rate of the DB model $U_{\mathrm{DB}}$, we make the common assumptions that all carrier flows are in steady state and that neighboring defects do not interact. In the case of $a-\mathrm{Si}: \mathrm{H}$, the second assumption is valid when the total density of defects $N_{T}$ is $<10^{16} \mathrm{~cm}^{-3}$, which is equivalent to an average separation of $d>300 \AA .^{2}$

The recombination and re-emission paths can then be divided into two parallel processes:

$$
T_{3}^{+}+e \leftrightarrow T_{3}^{0},
$$

consisting of $u_{1}, u_{3}, u_{5}$, and $u_{7}$, and

$$
T_{3}^{0}+e \leftrightarrow T_{3},
$$

consisting of $u_{2}, u_{4}, u_{6}$, and $u_{8}$. The flows $u_{1}$ to $u_{8}$ are given by $^{2}$

$$
\begin{aligned}
& u_{1}(E)=\bar{n}^{+} f^{+}(E) N_{t}(E), \quad u_{2}(E)=\bar{n}^{0} f^{0}(E) N_{t}(E), \\
& u_{3}(E)=e_{n}^{0}(E) f^{0}(E) N_{t}(E), \quad u_{4}(E)=e_{n}^{-}(E) f^{-}(E) N_{t}(E), \\
& u_{5}(E)=\bar{p}^{0} f^{0}(E) N_{t}(E), \quad u_{6}(E)=\bar{p}^{-} f^{-}(E) N_{t}(E), \\
& u_{7}(E)=e_{p}^{+}(E) f^{+}(E) N_{t}(E), \quad u_{8}(E)=e_{p}^{0}(E) f^{0}(E) N_{t}(E),
\end{aligned}
$$

where $N_{t}(E)$ is the density of defects equivalent to $E_{t}$ in Fig. 1. $f^{+}, f^{0}$, and $f^{-}$are the occupation fractions of the $T_{3}^{+}, T_{3}^{0}$, and $T_{3}$ states given by ${ }^{1,2}$

$$
\begin{aligned}
& f^{+}(E)=\frac{1}{1+\frac{e_{p}^{+}(E)+\bar{n}^{+}}{e_{n}^{0}(E)+\bar{p}^{0}}\left(1+\frac{e_{p}^{0}(E)+\bar{n}^{0}}{e_{n}^{-}(E)+\bar{p}^{-}}\right)}, \\
& f^{0}(E)=\frac{e_{p}^{+}(E)+\bar{n}^{+}}{e_{n}^{0}(E)+\bar{p}^{0}} f^{+}(E), \\
& f^{-}(E)=\frac{e_{p}^{+}(E)+\bar{n}^{+}}{e_{n}^{0}(E)+\bar{p}^{0}}\left(\frac{e_{p}^{0}(E)+\bar{n}^{0}}{e_{n}^{-}(E)+\bar{p}^{-}}\right) f^{+}(E) .
\end{aligned}
$$

The electron emission coefficients of $T_{3}^{0}$ and $T_{3}^{-}$are $e_{n}^{0}$ and $e_{n}^{-}$, while the hole emission coefficients of $T_{3}^{+}$and $T_{3}^{0}$ are $e_{p}^{+}$and $e_{p}^{02}$

$$
\begin{aligned}
& e_{n}^{0}(E)=\frac{\vec{n}_{0}^{+}}{2 \exp \left\{\beta\left[E_{F}-E_{t}(E)\right]\right\}}, \\
& e_{n}^{-}(E)=\frac{2 \vec{n}_{0}^{0}}{\exp \left\{\beta\left[E_{F}-E_{t}(E)-E_{u}\right]\right\}}, \\
& e_{p}^{+}(E)=2 \vec{p}_{0}^{0} \exp \left\{\beta\left[E_{F}-E_{t}(E)\right]\right\}, \\
& e_{p}^{0}(E)=\frac{1}{2} \overline{p_{0}} \exp \left\{\beta\left[E_{F}-E_{t}(E)-E_{u}\right]\right\},
\end{aligned}
$$

where $\beta=q / k T$ and $E_{F}$ is the equilibrium Fermi level. Equations (3)-(5) include the convenient definitions ${ }^{13}$

$$
\begin{aligned}
& \bar{n}^{+}=v_{\mathrm{th}} n \sigma_{n}^{+}, \quad \bar{n}^{0}=v_{\mathrm{th}} n \sigma_{n}^{0}, \quad \bar{p}^{0}=v_{\mathrm{th}} p \sigma_{p}^{0}, \quad \bar{p}=v_{\mathrm{th}} p \sigma_{p}^{-}, \\
& \bar{n}_{0}^{+}=v_{\mathrm{th}} n_{0} \sigma_{n}^{+}, \quad \bar{n}_{0}^{0}=v_{\mathrm{th}} n_{0} \sigma_{n}^{0}, \quad \bar{p}_{0}^{0}=v_{\mathrm{th}} p_{0} \sigma_{p}^{0}, \\
& \overline{p_{0}}=v_{\mathrm{th}} p_{0} \sigma_{p}^{-},
\end{aligned}
$$

where $v_{\text {th }}$ is the thermal velocity, $\sigma_{n}^{+}$and $\sigma_{n}^{0}$ (in $\mathrm{cm}^{2}$ ) are the electron capture cross sections of $T_{3}^{+}$and $T_{3}^{0}, \sigma_{p}^{0}$ and $\sigma_{p}^{-}$are the hole capture cross sections of $T_{3}^{0}$ and $T_{3}^{-}, n$ and $p$ are the steady-state electron and hole concentrations, and $n_{0}$ and $p_{0}$ are the equilibrium electron and hole concentrations. Note that we follow the common assumption that the capture cross sections are independent of energy. ${ }^{2,5-11}$

When the interaction between neighboring defects is negligible as assumed, $N_{t}(E)$ can be considered as a continuum of defects with discrete energy levels, and the total recombination rate $U_{\mathrm{DB}}$ can be given by integrating over energy,

$$
\begin{aligned}
U_{\mathrm{DB}}= & \int_{N_{t}(E)}\left(u_{1}+u_{2}-u_{3}-u_{4}\right) d E \\
= & \int_{N_{t}(E)}\left(u_{5}+u_{6}-u_{7}-u_{8}\right) d E \\
= & \int_{N_{t}(E)}\left[\bar{n}^{+} f^{+}(E)+\bar{n}^{0} f^{0}(E)-e_{n}^{0} f^{0}(E)\right. \\
& \left.-e_{n}^{-} f^{-}(E)\right] N_{t}(E) d E=\int_{N_{t}(E)} A(E) N_{t}(E) d E,
\end{aligned}
$$

where $A(E)$ represents the terms in square brackets.

Equation (7) is not usually applied in its full form for the calculation of DB recombination due to its mathematical complexity. In one simplification of this solution, ${ }^{2,5}$ a $\delta$ function is used for $N_{t}(E)$; recombination is only calculated for one energy level and integration is not required. Hubin et al. ${ }^{6}$ made an alternative simplification that avoided the integration as well as reduced the number of parameters. They assumed that the sample is sufficiently illuminated that the quasi-Fermi levels for traps lie outside $N_{t}(E)$. As a result, all defects act as recombination centers and the re-emission flows (i.e., $u_{3}, u_{4}, u_{7}$, and $u_{8}$ ) are negligible. As evident in Eq. (4), when the emission terms are neglected, the occupation fractions become independent of $E_{F}, E_{t}$, and $E_{u}$, giving 


$$
\begin{aligned}
& f_{\mathrm{scf}}^{+}=\frac{\bar{p} \bar{p}^{0}}{\bar{p}-\bar{p}^{0}+\bar{n}^{+} \bar{p}^{-}+\bar{n}^{+} n^{0}}, \quad f_{\mathrm{scf}}^{0}=\frac{\bar{n}^{+} \bar{p}^{-}}{\bar{p}^{-} \bar{p}^{0}+\bar{n}^{+} \bar{p}^{-}+\bar{n}^{+} \bar{n}^{0}}, \\
& f_{\mathrm{scf}}^{-}=\frac{\bar{n}^{+} \bar{n}^{0}}{\bar{p}-\bar{p}^{0}+\bar{n}^{+} \bar{p}^{-}+\bar{n}^{+} \bar{n}^{0}},
\end{aligned}
$$

and consequently, the solution to $U_{\mathrm{DB}}$ becomes analytic, ${ }^{6}$

$$
\begin{aligned}
U_{\mathrm{DB} . \mathrm{scf}} & =\int_{N_{t}(E)}\left(u_{1}+u_{2}\right) d E \\
& =\int_{N_{t}(E)}\left(u_{5}+u_{6}\right) d E \\
& =\left[\frac{\bar{n}^{+} \bar{p}^{-} \bar{p}^{0}+\bar{p}^{-} \bar{n}^{0} \bar{n}^{+}}{\bar{p}^{-} \bar{p}^{0}+\bar{n}^{+} \bar{p}^{-}+\bar{n}^{+} \bar{n}^{0}}\right] \int_{N_{t}(E)} N_{t}(E) d E=A_{\mathrm{scf}} N_{T} .
\end{aligned}
$$

The total density of states $N_{T}=\int N_{t}(E) d E$, and $A_{\text {scf }}$ represents the terms in the square brackets.

Being analytic and simple, Eq. (9) has been employed by several researchers of $a-\mathrm{Si}: \mathrm{H}$, yet the validity of its underlying assumption has not been investigated. While Hubin et $a l^{6}{ }^{6}$ stated that the quasi-Fermi levels for traps are those derived by Taylor and Simmons, ${ }^{13}$ the equations that describe these levels were not presented and are not trivial to derive. In the Sec. III, we derive the four quasi-Fermi levels for traps required to assess the validity of Eq. (9).

\section{DERIVATION OF THE QUASI-FERMI LEVELS FOR TRAPS}

As shown in Fig. 1, the electron and hole flows can be divided into two sets of processes. Note that many sets of processes will exist if the capture cross sections are not independent of energy. For $U_{\mathrm{DB} . \mathrm{scf}}$,

$$
\begin{aligned}
& T_{3}^{+} / T_{3}^{0} \text { process: } \bar{n}^{+}-e_{n}^{0}=\bar{p}^{0}-e_{p}^{+}, \\
& T_{3}^{0} / T_{3} \text { process: } \bar{n}^{0}-e_{n}^{-}=\bar{p}^{-}-e_{p}^{0} .
\end{aligned}
$$

Following Taylor and Simmons, ${ }^{13}$ the quasi-Fermi levels for traps correspond to the conditions where either $e_{p}^{+}, e_{n}^{0}, e_{p}^{0}$, or $e_{n}^{-}$is negligible. These four conditions can be expanded and rearranged to define the four quasi-Fermi levels for traps,

$$
\begin{aligned}
& e_{n}^{0}=\bar{n}^{+}+\bar{p}^{0}, \quad E_{t n}^{0}=E_{F}-\frac{1}{\beta} \ln \left(\frac{\bar{n}_{0}^{+}}{2 \bar{n}^{+}+2 \bar{p}^{0}}\right), \\
& e_{p}^{+}=\bar{n}^{+}+\bar{p}^{0}, \quad E_{t p}^{+}=E_{F}-\frac{1}{\beta} \ln \left(\frac{\bar{n}^{+}+\bar{p}^{0}}{2 \bar{p}_{0}^{0}}\right), \\
& e_{n}^{-}=\bar{n}^{0}+\bar{p}^{-}, \quad E_{t n}^{-}=E_{F}-E_{u}-\frac{1}{\beta} \ln \left(\frac{2 \bar{n}_{0}^{0}}{\bar{n}^{0}+\bar{p}^{-}}\right), \\
& e_{p}^{0}=\bar{n}^{0}+\bar{p}^{-}, \quad E_{t p}^{0}=E_{F}-E_{u}-\frac{1}{\beta} \ln \left(\frac{2 \bar{n}^{0}+2 \bar{p}^{-}}{\overline{p_{0}}}\right) .
\end{aligned}
$$

When the defect energy level being considered $E_{t}$ is below the quasi-Fermi level for trapped electrons, $E_{t n}^{0}$, for ex- (a)

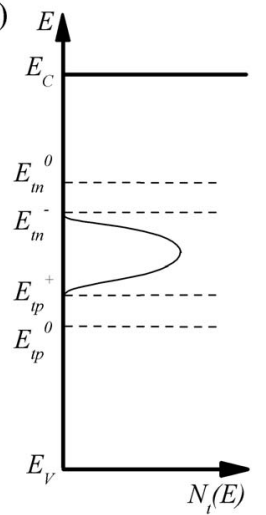

(b)

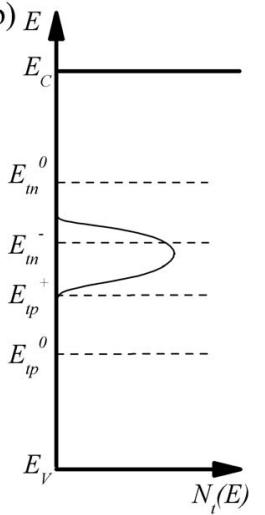

FIG. 2. (a) The key assumption is valid and $U_{\mathrm{DB} . \mathrm{scf}}$ is applicable when all the quasi-Fermi levels for traps are outside $N_{t}(E)$. (b) One of the quasiFermi level for traps is not outside $N_{t}(E)$; the assumption is invalid and $U_{\text {DB.scf }}$ should not be used.

ample, the probability of thermal re-emission to the conduction band via the corresponding flow (i.e., $u_{3}$ for this case) is negligible. This similarly applies to $E_{t n}^{-}$for electrons via $u_{4}$ and conversely for $E_{t p}^{+}$and $E t_{p}^{0}$ for holes via $u_{7}$ and $u_{8}$, respectively.

In the region where all of the conditions $E_{t}<E_{t n}^{0}, E_{t}$ $<E_{t n}^{-}, E_{t}>E_{t p}^{+}$, and $E_{t}>E_{t p}^{0}$ are fulfilled simultaneously, all thermal re-emission flows are negligible and any defect level within this region will act as a recombination center and not as a trap. When all four quasi-Fermi levels for traps lie outside the entire distribution of states $N_{t}(E)$, all the defect levels act as recombination centers [Fig. 2(a)]. In such a case, the key assumption is valid and thus it is appropriate to use $U_{\text {DB.scf. }}$

On the other hand, when not all of the quasi-Fermi levels for traps lie outside the distribution of $N_{t}(E)$, then the conditions for the key assumption are not met. In Fig. 2(b), for example, all defects between $E_{t n}^{-}$and $E_{t p}^{+}$are recombination centers but only some defects between $E_{t n}^{-}$and $E_{t n}^{0}$ are recombination centers. Since not all of $N_{t}(E)$ are recombination centers, the assumption is invalid and $U_{\mathrm{DB} \text {.scf }}$ should not be used.

\section{VALIDITY OF THE SIMPLE CLOSED-FORM SOLUTION}

The suitability of using the quasi-Fermi levels for traps to validate $U_{\mathrm{DB} . s c f}$ is determined by comparing the mathematical error between $U_{\mathrm{DB} . s c f}$ and $U_{\mathrm{DB}}$ as well as their energy dependence. By graphically comparing the quasi-Fermi levels for traps with the energies at which those differences occur, we can determine if the quasi-Fermi levels for traps are actually useful for establishing the boundaries for the applicability of $U_{\text {DB.scf. }}$.

To demonstrate this we present below an example using a Gaussian function for $N_{t}(E)$ and typical values ${ }^{2,5,14}$ for the variables in Eqs. (5) and (6) (listed in the Appendix). This method has also been applied to a variety of $N_{t}(E)$ and variables, and the results are similar to the examples we present here.

Figure 3 graphs the energy dependence of $U_{\mathrm{DB} . s c f}$ and $U_{\mathrm{DB}}$. The gray area in Fig. 3(b) graphically represents 
(a)

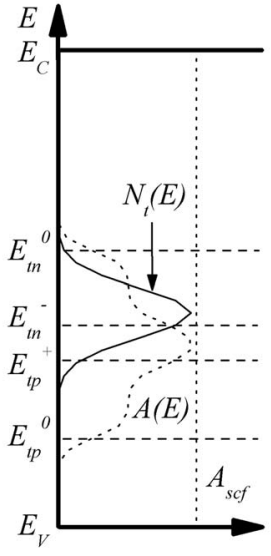

(b)

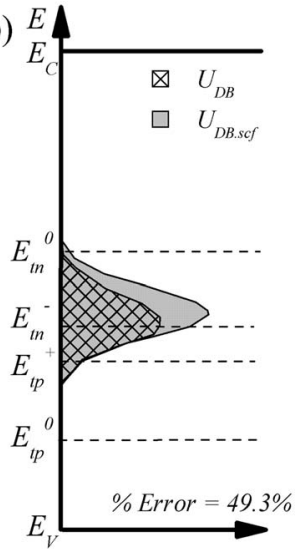

FIG. 3. Energy dependence of $U_{\mathrm{DB} . s c f}$ and $U_{\mathrm{DB}}$ and the corresponding quasiFermi levels for traps in a case where the quasi-Fermi levels for traps do not lie outside the distribution of $N_{t}(E)$. (a) $N_{t}(E), A(E)$, and its corresponding function of $A_{\text {scf. }}$ (b) The areas under the curves give the numerical and the simple solutions to the total recombination in the DBs.

$U_{\text {DB.scf: }}$ by multiplying the functions of $N_{t}(E)$ and $A_{\text {scf }}$ in Fig. 3(a) with $v_{\text {th }}$ as in Eq. (9), we obtain the function $\left(u_{1}+u_{2}\right)$, and the gray area under this function is equivalent to the integral (i.e., $\left.U_{\mathrm{DB} . s c f}\right)$. Similarly the cross-hatched area graphically represents $U_{\mathrm{DB}}$ : multiplying the functions of $N_{t}(E)$ and $A(E)$ in Fig. 3(a) with $v_{\text {th }}$ as in Eq. (7) gives $\left(u_{1}\right.$ $\left.+u_{2}-u_{3}-u_{4}\right)$ in Fig. 3(b), and the integral of this gives the numerical solution of $U_{\mathrm{DB}}$. In Fig. 3, we can clearly see that the discrepancy between gray and cross-hatched areas (i.e., $U_{\mathrm{DB} . s c f}$ and $U_{\mathrm{DB}}$, respectively) occurs precisely where the quasi-Fermi levels for traps do not lie outside the distribution of $N_{t}(E)$.

The percentage error of using $U_{D B . s c f}$ rather than $U_{D B}$ is given by

$$
\text { \%error }=\frac{U_{\mathrm{DB}}-U_{\mathrm{DB} . \mathrm{scf}}}{U_{\mathrm{DB}}},
$$

and is about $49.3 \%$ (global error) in this case, showing the inadequacy of the simple model when the key assumption is not valid.

\section{A. Effect of illumination}

According to Hubin et al., ${ }^{6}$ the key assumption generally is valid when the illumination is sufficiently high and the excess carrier density is also high, but eventually becomes invalid as the illumination decreases. Therefore, we can confirm the predictions of Hubin et al..$^{6}$ on the effect of excess carrier generation.

In Fig. 4, we show the effect of (a) low $(\Delta n=\Delta p$ $\left.=10^{11} \mathrm{~cm}^{-3}\right)$ and (b) high illumination $(\Delta n=\Delta p=5$ $\times 10^{14} \mathrm{~cm}^{-3}$ ) using $N_{t}(E)$ as in Fig. 3(a). In Fig. 4(a) we can see that the assumption is invalid at low illumination due to the fact that the quasi-Fermi levels for trapped electrons and holes are close to each other and do not lie outside $N_{t}(E)$. The error of $U_{\mathrm{DB} . s c f}$ is very high, at $51.3 \%$. In Fig. 4(b) we show the case when the illumination is high enough such that the assumption is valid. The splitting between the quasi- (a)

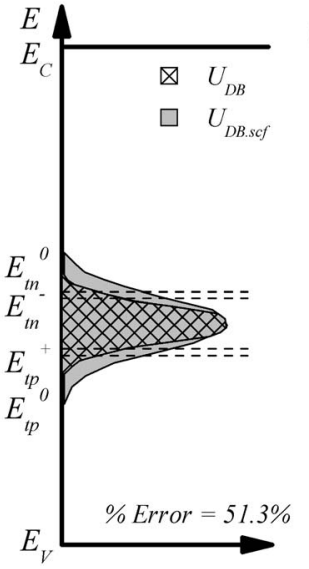

FIG. 4. (a) At low illumination the quasi-Fermi levels for traps are very close in energy; the assumption is invalid and the use of $U_{\mathrm{DB} . s c f}$ will lead to large errors. (b) At sufficiently high illumination, all the quasi-Fermi levels for traps lie outside the distribution of $N_{t}(E)$; the areas under the curves are almost the same, demonstrating that it is appropriate to use the $U_{\mathrm{DB} . s c f}$ solution.

Fermi levels for traps is very large and outside $N_{t}(E)$, making the assumption valid and reducing the error of the simple model to $0.4 \%$.

\section{B. Effect of other parameters}

The other parameters such as $E_{u}$ and the capture cross sections will change the values of the quasi-Fermi levels for traps and subsequently influence the illumination level at which the key assumption becomes invalid. Increasing the value of $E_{u}$, for example, will shift the quasi-Fermi levels for traps $E_{t n}^{-}$and $E_{t p}^{0}$ to lower energies. This will narrow the region in which all the defect levels act as recombination centers, thus increasing the error. For example, a change in $E_{u}$ from $0.1 \mathrm{eV}$ [Fig. 4(b)] to $0.35 \mathrm{eV}$ [Fig. 5(a)] increases the error from $0.4 \%$ to $16.7 \%$. A higher illumination is needed $\left(\Delta n=\Delta p=10^{18} \mathrm{~cm}^{-3}\right)$ for the key assumption to be valid for this value of $E_{u}$ [Fig. 5(b)], and the error reduces to $0.5 \%$. Changing the values of the capture cross sections will also have an effect, but it is generally less substantial.

(a)

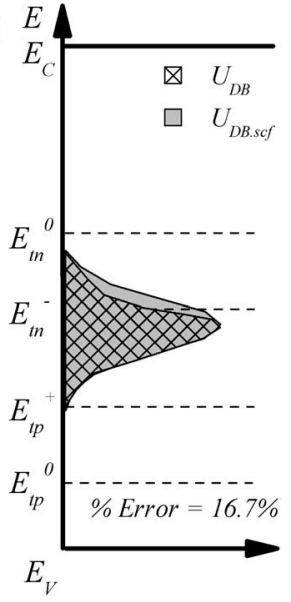

(b)

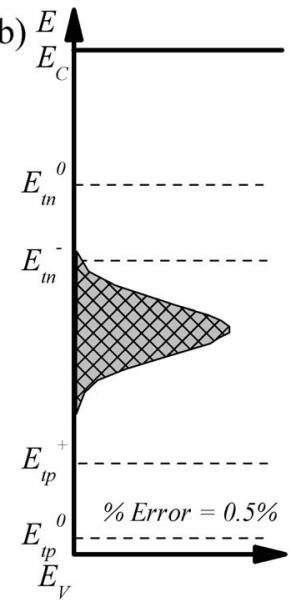

FIG. 5. The effect of increasing $E_{u}$. (a) $E_{t n}^{-}$and $E_{t p}^{+}$will be closer in energy and the region of validity will be smaller. (b) A higher illumination is needed to make the key assumption valid. 


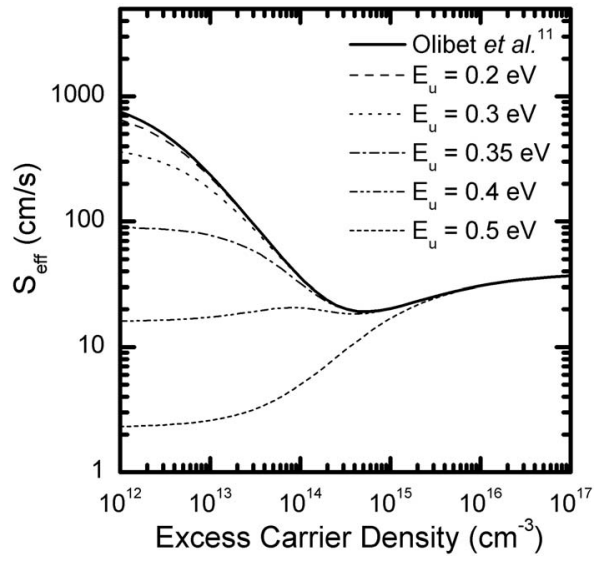

FIG. 6. An example highlighting the effect of $E_{u}$ on the modeling of recombination at an $a-\mathrm{Si}: \mathrm{H}(\mathrm{i}) / c-\mathrm{Si}(\mathrm{n})$ interface. The effective surface recombination velocity $\left(S_{\text {eff }}\right)$ using $U_{\text {DB.scf }}$ as applied by Olibet et al. (Ref. 11) is compared to the $S_{\text {eff }}$ calculated from $U_{\mathrm{DB}}$ for various values of $E_{u}$.

\section{DISCUSSION}

The simple closed form developed by Hubin et al. ${ }^{6}$ $\left(U_{\text {DB.scf }}\right)$ has been applied to various $a$-Si:H structures, assuming, without verification, that the quasi-Fermi levels for traps were outside $N_{t}(E)$. As $N_{t}(E)$ and the values of $E_{u}$ and $E_{F}$ of the previous applications are unknown, the use of $U_{\text {DB.scf }}$ cannot be assessed. However, we can evaluate the impact of a range of possible values for $E_{u}$. The example given in Fig. 6 is a $60 \Omega \mathrm{cm} n$-type $c$-Si wafer that has 40 $\mathrm{nm} a$-Si:H(i) on both its surfaces to achieve surface passivation. Olibet $e t a l{ }^{11}$ adapted $U_{\mathrm{DB} . s c f}$ to model the surface recombination velocity at the interface between the $a$-Si:H and the $c$-Si wafer and noted the discrepancy between their experimental data and their modeling at low carrier densities. We reproduce the modeling as the solid black line in Fig. 6.

Since the simple model cannot account for a possible range of values of $E_{u}$, we have used the more detailed numerical solution for $U_{\mathrm{DB}}$ and adapted it to determine the surface recombination velocity for different values of $E_{u}$. The results are graphed in Fig. 6 as well.

By comparing with the simple solution, we can see for this case that when $E_{u}$ is between 0 and $0.2 \mathrm{eV}$, the use of the simple closed-form solution is reasonable. As $E_{u}$ increases, however, the error can increase to several orders of magnitude. For example, at $\Delta n=\Delta p=10^{12} \mathrm{~cm}^{-3}, \quad S_{\text {eff }}$ is $\sim 800 \mathrm{~cm} / \mathrm{s}$ for $E_{u}=0.2 \mathrm{eV}$; meanwhile at $E_{u}=0.35 \mathrm{eV}, S_{\text {eff }}$ is much lower at $\sim 90 \mathrm{~cm} / \mathrm{s}$. The illumination level (the excess carrier density) at which the assumption becomes invalid also increases.

From an observation of the experimental data in Ref. 11, the $E_{u}$ of the sample is $\sim 0.3 \mathrm{eV}$, although additional parameters need to be known before this can be verified. However, fitting the experimental data to $E_{u}=0.3 \mathrm{eV}$ may lead to changes in the fitted values for the capture cross sections, for example, and thus fitting using the $U_{\mathrm{DB} . s c f}$ solution would be incorrect. This case demonstrates how the application of $U_{\text {DB.scf }}$ can be incorrect and the importance of ensuring the validity of the key assumption.

The approximate $U_{\mathrm{DB} . s c f}$ solution reduces the number of parameters that need to be known and simplifies the calculations enormously. However, these very same parameters [i.e., $N_{t}(E), E_{u}$, and $E_{F}$ ] need to be known in order to determine whether it is suitable and valid to use. While estimates for their values can be made from a theorectical ${ }^{2,5,14}$ or an experimental ${ }^{14,15}$ basis, they cannot be ignored without consideration.

It is important, however, to note that the same parameters needed for calculating the quasi-Fermi levels for traps enable the calculation of $U_{\mathrm{DB}}$ using the numerical solution. Consequently, if one calculates the quasi-Fermi levels for traps and finds that it is inappropriate to use the approximate $U_{\mathrm{DB} . s c f}$, then $U_{\mathrm{DB}}$ can be used. While this solution has been derived for $a-\mathrm{Si}: \mathrm{H}$, it is not exclusive to it and can be applied to any recombination mechanism with the same statistics.

\section{CONCLUSIONS}

Hubin et al. ${ }^{6}$ greatly simplified the calculations for the recombination in the DBs, where there are three charge states and two energy levels, by making several assumptions leading to a simple closed-form solution, but they did not explicitly define its limitations. In this paper we have derived, based on the recombination statistics for this system, the four quasi-Fermi levels for traps that need to be considered. By comparing $U_{\mathrm{DB} \text {.scf }}$ with the numerical solution of $U_{\mathrm{DB}}$, we have shown how these quasi-Fermi levels for traps can properly define the limitations of $U_{\mathrm{DB} . s c f}$ and how they are affected by the various parameters of the material. We conclude that it is only appropriate to use $U_{\mathrm{DB} \text {.scf }}$ when both the quasi-Fermi levels for trapped electrons $E_{t n}^{0}$ and $E_{t n}^{-}$are higher in energy than the complete distribution of $N_{t}(E)$ and both the quasi-Fermi levels for trapped holes $E_{t p}^{+}$and $E_{t p}^{0}$ are lower in energy than the complete distribution of $N_{t}(E)$.

When the above conditions are not satisfied, the application of $U_{\mathrm{DB} . s c f}$ using Eqs. (6), (8), and (9) can lead to large errors in the calculation of recombination in the DBs. Therefore, it is important to calculate the values of the quasi-Fermi levels for traps in Eq. (11) with the knowledge of the additional parameters $N_{t}(E), E_{u}$, and $E_{F}$ to check the validity of the key assumption and determine the appropriateness of using $U_{\mathrm{DB} . s c f}$. However, these very same parameters can be applied to the numerical solution [Eqs. (3)-(7)] to provide a complete and more accurate determination of $U_{\mathrm{DB}}$. Given the capabilities of modern computing, the additional calculations that arise from using the numerical solution of $U_{\mathrm{DB}}$ should

TABLE I. List of modeling parameters.

\begin{tabular}{lccccc}
\hline \hline Parameter & Figure 3(b) & Figure 4(a) & Figure 4(b) & Figure 5(a) & Figure 5(b) \\
\hline$\Delta n=\Delta p\left(\mathrm{~cm}^{-3}\right)$ & $1 \times 10^{13}$ & $1 \times 10^{11}$ & $5 \times 10^{14}$ & $5 \times 10^{14}$ & $1 \times 10^{18}$ \\
$E_{u}(\mathrm{eV})$ & 0.35 & 0.1 & 0.1 & 0.35 & 0.35 \\
\hline \hline
\end{tabular}


not be a problem, and it would be advantageous to avoid any errors that would arise from the use of $U_{\mathrm{DB} . s c f}$.

\section{APPENDIX: MODELING PARAMETERS}

This section lists the parameters used for the modeling calculations for Figs. 3-5. The parameters common to all figures were $n_{0}=p_{0}=10^{10} \mathrm{~cm}^{-3}, \quad v_{\mathrm{th}}=10^{7} \mathrm{~cm} / \mathrm{s}, E_{C}-E_{V}$ $=1.9 \mathrm{eV}, \quad E_{F}=0.9 \mathrm{eV}, \quad \sigma_{P}^{0}=\sigma_{n}^{0}=10^{-16} \mathrm{~cm}^{2}, \quad \sigma_{p}^{-}=\sigma_{n}^{+}=5$ $\times 10^{-15} \mathrm{~cm}^{2}$, while $T=300 \mathrm{~K}$. The numerical integration was done at $0.05 \mathrm{eV}$ intervals. Table I lists the modeling parameters that were varied for each figure.

The Gaussian function for $N_{t}(E)$ is given by

$$
N_{t}(E)=10^{15} \exp \left[-\frac{(E-0.85)^{2}}{0.02}\right] \text {. }
$$

The total density of states $N_{T}$ resulting from the numerical integration of this function is $2.51 \times 10^{14} \mathrm{~cm}^{-3}$.
${ }^{1}$ H. Okamoto and Y. Hamakawa, Solid State Commun. 24, 23 (1977).

${ }^{2}$ F. Vaillant and D. Jousse, Phys. Rev. B 34, 4088 (1986).

${ }^{3}$ W. Shockley and W. T. J. Read, Phys. Rev. 87, 835 (1952)

${ }^{4}$ J. G. Simmons and G. W. Taylor, Phys. Rev. B 4, 502 (1971).

${ }^{5}$ F. Wang and R. Schwarz, Phys. Rev. B 52, 14586 (1995).

${ }^{6}$ J. Hubin, A. V. Shah, and E. Sauvain, Philos. Mag. Lett. 66, 115 (1992)

${ }^{7}$ G. Masini, D. Fischer, J. Hubin, F. Palmer, and A. V. Shah, Proceedings of the First WCPEC (IEEE, Hawaii, 1994).

${ }^{8}$ J. Hubin, A. V. Shah, E. Sauvain, and P. Pipoz, J. Appl. Phys. 78, 6050 (1995).

${ }^{9}$ J. Hubin and A. V. Shah, Philos. Mag. B 72, 589 (1995).

${ }^{10}$ J. Merten, J. M. Asensi, C. Voz, A. V. Shah, R. Platz, and J. Andreu, IEEE Trans. Electron Devices 45, 423 (1998).

${ }^{11}$ S. Olibet, E. Vallat-Sauvain, and C. Ballif, Phys. Rev. B 76, 035326 (2007).

${ }^{12}$ A. Rose, Concepts in Photoconductivity and Allied Problems (Interscience Publishers, New York, 1963).

${ }^{13}$ G. W. Taylor and J. G. Simmons, J. Non-Cryst. Solids 8-10, 940 (1972).

${ }^{14}$ R. A. Street, Hydrogenated Amorphous Silicon (Cambridge University Press, Massachusetts, 1991).

${ }^{15}$ J. Kočka, C. E. Nebel, and C. D. Abel, Philos. Mag. B 63, 221 (1991). 